\title{
Defibrillation Threshold
}

National Cancer Institute

\section{Source}

National Cancer Institute. Defibrillation Threshold. NCI Thesaurus. Code C101236.

The lowest energy where a defibrillator device terminates an arrhythmia in a particular subject. (ACC) 